\title{
Comparison of volumetric and bidimensional measurement of the thymic gland to determine interobserver variability
}

\author{
๑Özlem Demircioğlu, (Canan Çimşit, @Nuri Çagatay Çimşit \\ Marmara University Pendik Education and Research Hospital, Department of Radiology, Istanbul, Turkey
}

Cite this article as: Demircioğlu Ö, Çimşit C, Çimşit NÇ. Comparison of volumetric and bidimensional measurement of the thymic gland to determine interobserver variability. Anatolian Curr Med J 2022; 4(1); 51-54.

\begin{abstract}
Aim: Thymic measurements should be interpreted relative to normal measurement values according to age groups and gender. Thymic gland measurement may vary between radiologists. In our study we wanted to determine the interobserver variability of thymic size measurements on CT by comparing volumetric calculations to commonly used bidimensional measurements.

Material and Method: The chest CT scans of 156 patients who had any oncological problems or disorders influencing the thymic size were retrospectively evaluated. The shape (quadrilateral or triangular), anteroposterior (AP) and transverse (TR) diameters, as well as the thickness of each lobe and thymus volume were measured independently by two radiologists. Interobserver variability was determined according to these parameters by Pearson correlation test.

Results: The shape of the thymic gland was triangular in $72 \%$ (n:113) and quadrilateral in $28 \%$ (n: 43) of all subjects. According to the results of the correlation test, a moderate relationship in terms of AP length, a weak relationship in terms of TR length, a negligible relationship in terms of right lobe thickness and a weak relationship in terms of left lobe thickness were obtained, but a very strong relationship was found between the two radiologists for volumetric measurements.

Conclusions: Volumetric measurements can be used as a basis for thymic imaging, reducing the differences among radiologists, and unnecessary and advanced examinations can be avoided.
\end{abstract}

Keywords: Thymus gland, volumetric measures, bidimensional measures, computerized tomography

\section{INTRODUCTION}

The thymus differs in size, shape and density in each individual. The thymic gland changes with time, reaching its maximum size in puberty due to increased sex hormones during adolescence and then shrinking in adulthood as a result of involution by fat tissue $(1,2)$. In addition to these physiological changes, gender, physical differences, smoking, stress, and severe illnesses/disorders and treatments affecting the thymus make it challenging for radiologists to determine if a gland is "normal" using cross-sectional imaging (3). As the thymus appears in a variety of shapes, including pyramidal, triangular, arrow head, and quadrilateral, any space-occupying lesion can be confused with the gland (4-7). Consequently, there can be misdiagnosis or failure to diagnose normal from abnormal results in follow-up examinations, biopsies and thymectomies $(8,9)$.

Multi-detector row Computed Tomography (MDCT) scanners provide novel information about the thymus due to contiguous thin slices, multiplanar reformats, 3D quantification and volumetrics. CT characteristics, bidimensional measurements and volumetric calculations of the thymus have been reported in the literature $(10,11)$. Measurements on CT are subject to interobserver variability and there are ongoing studies defining the intra- and interobserver variability of thickness, diameter and density values (12). This study aimed to evaluate the interobserver variability of thymic bidimensional and volumetric measurements of chest CTs.

\section{MATERIAL AND METHOD}

The study was approved by Marmara University NonInterventional Clinical Researchs Ethics Committee (Date: 12.04.2019, Decision No: 09.2019.350) and by the relevant committee or the Helsinki Declaration of Principles. 


\section{Patient Selection}

In this study the chest CT scan of patients who were admitted to our hospital for any reason other than oncological problems between March 2018 and July 2020 were evaluated retrospectively. Any known disorders influencing the size of the thymus, patients with mediastinal mass, hematoma, patients undergoing chemotherapy and patients with thymus showing complete fat replacement were excluded (n:100).

\section{Equipment and CT Examination}

All examinations were performed on a 256 slice scanner (Somatom Definition Flash, Siemens, Erlangen, Germany) with the following scan parameters: CARE Dose $4 \mathrm{D}$ for automatic exposure control for $120 \mathrm{kV}$ and $50 \mathrm{mAs}$, $5 \mathrm{~mm}$ slices acquired with $128 \times 0.6 \mathrm{~mm}$ setting, 0.5 pitch and 0.28 second rotation time. FOV was adjusted to patient size, a $512 \times 512$ matrix was used and the mean scan time was 4.2 seconds. Additional $0.75 \mathrm{~mm}$ axial reconstructions were generated as well as $10 \mathrm{~mm}$ multi-planar reformat (MPR) images in sagittal, coronal and axial planes for every examination. All patients received a bolus of 80 to 100 $\mathrm{ml}$ of intravenous contrast matter at a rate of 3 to $4 \mathrm{ml} / \mathrm{s}$. The enhanced CT scan commenced 60 seconds after the administration of the contrast medium.

\section{Image Interpretation}

All patient data were examined separately on a PACS system (Novapacs, Novarad Corporation, USA) by two radiologists with 6 and 17 years of expertise, respectively, in radiology, and each radiologist documented their findings. The radiologists were free to adjust the window level and width and review sagittal and coronal images as well. The shape of the thymus was classified in two groups as quadrilateral or triangular (Figure 1). The axial CT images that provided the largest images of the thymus in terms of overall size and length were determined. The anteroposterior (AP) and transverse (TR) diameters of the gland were measured on this axial image from the thickest point of the gland using the caliper tool on the PACS system (Figure 2). The thickness of each lobe was also measured. The axial CT image with the largest area of each thymic lobe was selected for this (Figure 3).

Thymic gland volumes were measured using a software application (syngo.CTPulmo 3D, Siemens, Erlangen) after the chest CT images of patients were transferred to the Siemens Syngo Multimodality Workplace. Automated volume calculation was developed on the basis of $3 \mathrm{D}$ active contouring. Both radiologists manually traced the contour of the thymus on each CT slice and created this $3 \mathrm{D}$ contour to determine the thymic volume (Figure 4). Each case had an average of five slices (3-7). Volume was determined by multiplying the sum of all slices with the 3D image reconstruction and volume-rendering tool.
The processing time was approximately 5 min per case. Results of each case in terms of volume were summarized by arithmetic mean and corresponding standard deviation as well as minimum and maximum values.

\section{Statistical Analysis}

The data of all patients were evaluated in SPSS (Statistical Package for Social Sciences, Chicago, IL, USA) system version 21. Data distribution analysis of volume and all measurement values were performed. As a result of this analysis, normal distribution was determined for all parameters. Then, a non-parametric bi-variate correlation test (Pearson correlation test) was used to determine the correlation between the two radiologists. According to the results, $\mathrm{p}$ and $\mathrm{r}$ values were calculated. $\mathrm{p}<0.05$ was considered statistically significant. $r$ values were considered as having a very strong relationship for +.70 or higher, a strong relationship for +.40 to +.69 , a moderate relationship for +.30 to .39 , a weak relationship for .20 to .29 and no or negligible relationship for 0 to .19

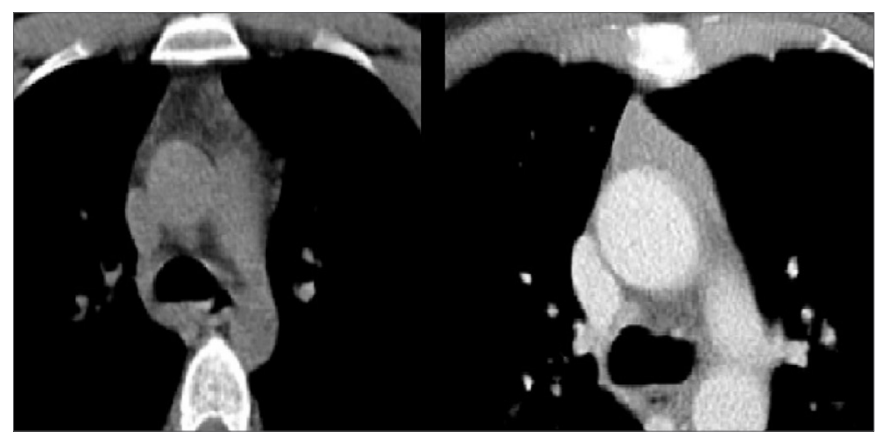

Figure 1. CT image example of quadrilateral (a) and triangular (b) shapes of the thymus.

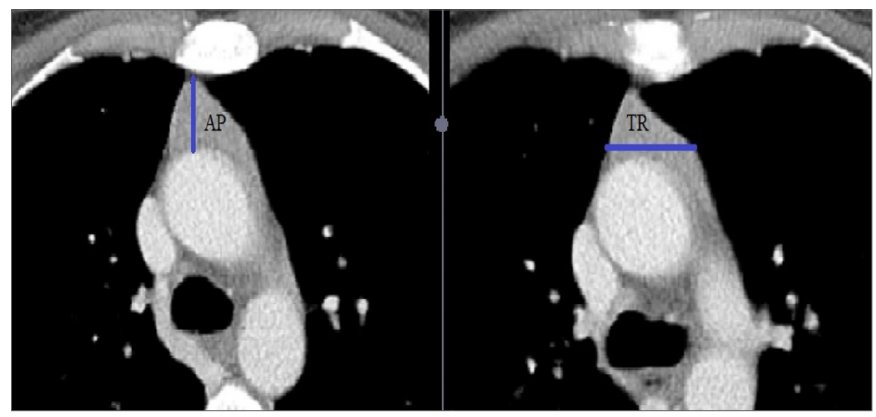

Figure 2. The measurements of anteroposterior (AP) and transverse (TR) diameters from the thickest point of the gland on axial CT images.

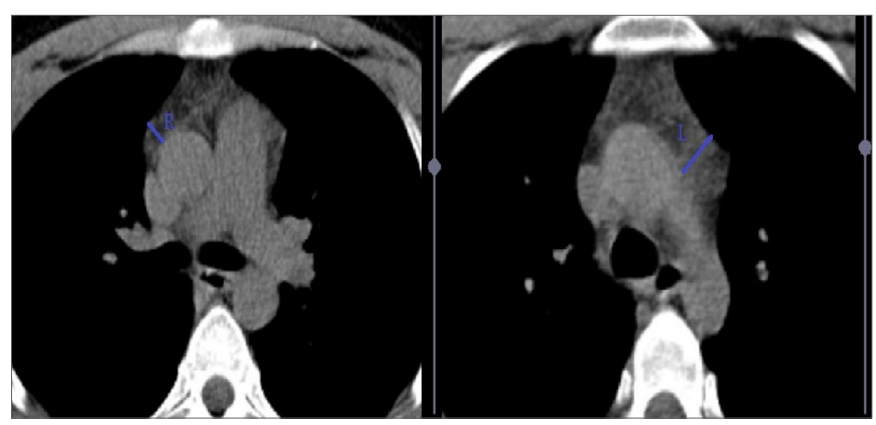

Figure 3. The axial CT image with the largest area of thymic lobe was selected for measuring the thickness of each lobes. 


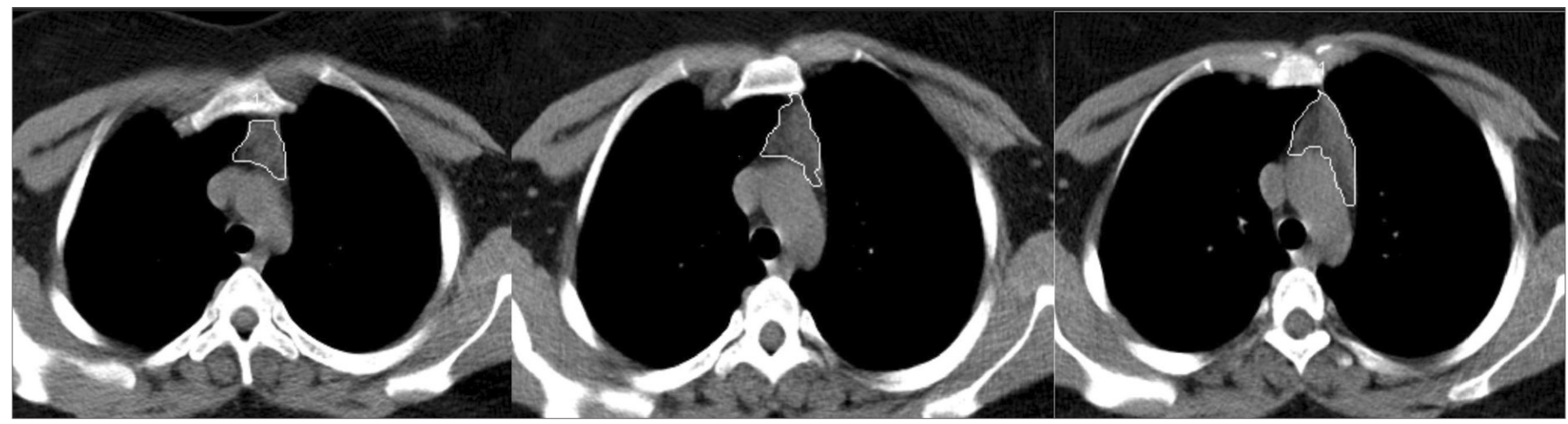

Figure 4. The volumetric contours of the thymus on CT slice.

\section{RESULTS}

One hundred and fifty-six patients (67 females, 89 males) were included in the study. The median age was 33.2 years (range: 17-79 years). The shape of the thymus gland was triangular in $72 \%$ (n:113) and quadrilateral in 28\% (n: 43) of all subjects enrolled in the study.

The thymic size measurements of the two radiologists including the AP and transverse diameters of the thymus, thickness of each lobe and volume of the gland are summarized in Table 1. According to the results of the Pearson correlation test, although a statistically significant relationship was found among the radiologists in all measurement parameters, a moderate relationship in terms of AP length $(\mathrm{r}=0,376)$, weak relationship in terms of TR length $(r=0,204)$, negligible relationship in terms of right lobe thicknes $(r=0,184)$ and weak relationship in terms of left lobe thicknes $(r=0,299)$ were obtained. In addition, a very strong relationship was found between the two radiologists for volumetric measurements $(\mathrm{r}=0,957) . \mathrm{p}$ and $\mathrm{r}$ values are given in Table 2.

\section{DISCUSSION}

In our study, the interobserver variability in thymic size measurement on CT was investigated by comparing two radiologists' volumetric calculations to commonly used bidimensional measurements. To the best of our knowledge, this study is the first to determine the interobserver variability of thymic volumetric calculations on CT compared to the variability of routinely used bidimensional measurements such as the transverse and AP diameters and thickness of each lobe. Our results showed a very strong relationship between the volumetric measurements of the two radiologists, which were more accurate than bidimensional measurements for thymic evaluation.

Each radiologist determined the image to be measured independently when performing bidimensional measurements. When the data were obtained, it was seen that only $40 \%$ of the selected axial images were in agreement. Araki et al. (3) found that the rate at which two radiologists chose the same image was $49 \%$,

\begin{tabular}{|c|c|c|c|c|c|}
\hline Radiologist & Tr length (mm) & AP length (mm) & Right Lobe Thickness (mm) & LeftLobe Thickness (mm) & Volume $(\mathrm{cm} 3)$ \\
\hline \multicolumn{6}{|l|}{ Radiologist 1} \\
\hline $\mathrm{N}$ & 156 & 156 & 156 & 156 & 156 \\
\hline Minimum & 8.80 & 10.40 & 3.10 & 3.70 & 4.00 \\
\hline Maximum & 47.00 & 56.00 & 22.10 & 19.00 & 30.60 \\
\hline Mean & 18.6878 & 19.4115 & 7.5199 & 8.1808 & 12.8671 \\
\hline Std. Deviation & 4.97460 & 6.65665 & 3.11039 & 2.60013 & 5.79009 \\
\hline \multicolumn{6}{|l|}{ Radiologist 2} \\
\hline $\mathrm{N}$ & 156 & 156 & 156 & 156 & 156 \\
\hline Minimum & 8.20 & 11.70 & 3.30 & 5.10 & 4.40 \\
\hline Maximum & 43.90 & 58.70 & 21.90 & 23.40 & 32.20 \\
\hline Mean & 22.9564 & 28.1256 & 9.3942 & 10.2538 & 12.9503 \\
\hline Std. Deviation & 6.72529 & 8.23478 & 2.85086 & 2.87455 & 5.67049 \\
\hline
\end{tabular}

Table 2. $r$ and $p$ values of thymus size and volumetric measurements of the thymus.

\begin{tabular}{|lccccc|} 
Group Variable & $\begin{array}{c}\text { AP } \\
\text { length }\end{array}$ & $\begin{array}{c}\text { Tr } \\
\text { length }\end{array}$ & $\begin{array}{c}\text { Right } \\
\text { Lobe }\end{array}$ & $\begin{array}{c}\text { Left } \\
\text { Lobe }\end{array}$ & Volume \\
\hline $\begin{array}{l}\text { Pearson } \\
\text { Correlation }(\mathrm{r})\end{array}$ & 0.376 & 0.204 & 0.184 & 0.299 & 0.957 \\
p value & $<0.001$ & 0.011 & 0.022 & $<0.001$ & $<0.001$ \\
\hline
\end{tabular}

Table 3. Thymus shape according to gender.

\begin{tabular}{|lcc|}
\hline & Triangular (n) & Quadrilateral (n) \\
\hline Male (89) & $65(73 \%)$ & $24(27 \%)$ \\
Female (67) & $48(72 \%)$ & $19(28 \%)$ \\
\hline
\end{tabular}


which is similar to our results. In their study, it was stated that there would be more agreement between the measurements if the image sections were chosen independently by the radiologists. The independence of the selected image sections in our study resulted in more consistent bidimensional measurements compared to other studies.

McErlean et al. (13) mentioned the importance of selecting the image by both of the radiologists before hand and suggested that measurements should be made in consecutive series in order to minimize measurement differences. We believe that since the volumetric measurements included the measurement of all images containing the thymus, the incompatibility between the radiologists' preferences for the selected image is unimportant; thus, unnecessary advanced examinations will be prevented. Our suggestion is in line with Simanovsky et al. (10), who performed a study that included volumetric measurements of the thymus with the aim of preventing unnecessary advanced examinations and recurrent radiation exposure.

On the other hand, Araki et al. (7) noted increased coherence between radiologists performing measurements on pre-determined images in patients with increased left lobe thickness. We suggest that when volumetric measurements are preferred over image selection, no matter whether it is one image or consecutive images, results would not be affected by the shape of the thymus or the status of thymus lobes.

Considering the physiological and pathological processes that result in the thymus shrinking or enlarging, which are mentioned both in our study and in the literature, we suggest that volumetric measurements would be more accurate than bidimensional measurements in terms of interobserver variability.

Our study has some limitations. First, this was a retrospective study and second, the number of patients included in the study was not large. Future studies with more subjects are needed to systematize the volumetric measurement method and hence contribute to the literature.

\section{CONCLUSION}

Interobserver variability of thymic bidimensional measurements was determined by our study as well as by previous studies. Our study suggests that changes in thymic volume can be accurately mapped with the noninvasive method of volumetric measurement, which can be used as a basis for thymic imaging and can reduce the differences among radiologists, thus avoiding unnecessary advanced examinations.

\section{ETHICAL DECLARATION}

Ethics Committee Approval: The study was approved by Marmara University Non-Interventional Clinical Researchs Ethics Committee (Date: 12.04.2019, Decision No: 09.2019.350)

Informed Consent: Because the study was designed retrospectively, no written informed consent form was obtained from patients.

Referee Evaluation Process: Externally peer-reviewed.

Conflict of Interest Statement: The authors have no conflicts of interest to declare.

Financial Disclosure: The authors declared that this study has received no financial support.

Author Contributions: All of the authors declare that they have all participated in the design, execution, and analysis of the paper, and that they have approved the final version.

\section{REFERENCES}

1. Aw D, Silva AB, Palmer DB. Immunosenescence: emerging challenges for an ageing population. Immunology 2007; 120: 435-46.

2. Elmore S. Enhanced histopathology evaluation of thymus. Toxicol Pathol 2006; 34: 656-65.

3. Araki T, Nishino M, Gao W, et al. Normal thymus in adults. appearance on CT and associations with age, sex, BMI and smoking. Eur Radiol 2016; 26: 15-24.

4. Baron R, Lee J, Sagel S, et al. Computed tomography of the normal thymus. Radiology 1982; 142: 121-5.

5. Francis I, Glazer G, Bookstein F, et al. The thymus: reexamination of age-related changes in size and shape. Am J Roentgenol 1985; 145: 249-54.

6. Ackman JB, Kovacina B, Carter BW, et al. Sex difference in normal thymic appearance in adults 20-30 years of age. Radiology 2013; 268: 245-53.

7. Araki T, Sholl LM, Gerbaudo VH, et al. Imaging characteristics of pathologically proven thymic hyperplasia: identifying features that can differentiate true from lymphoid hyperplasia. AJR Am J Roentgenol 2014; 202: 471-8.

8. Singla S, Litzky LA, Kaiser LR, et al. Should asymptomatic enlarged thymus glands be resected? J Thorac Cardiovasc Surg 2010; 140: 977-83.

9. Ackman JB, Mino-Kenudson M, Morse CR. Nonsuppressing normal thymus on chemical shift magnetic resonance imaging in a young woman. J Thorac Imaging 2012; 27: 196-8.

10. Simanovsky N, Hiller N, Loubashevsky N, et al. Normal CT characteristics of the thymus in adults. Eur J Radiol 2012; 81: 3581-6.

11. Prodhomme O, Seguret F, Martrille L, et al. Organ volume measurements: comparison between MRI and autopsy findings in infants following sudden unexpected death. Arch Dis Child Fetal Neonatal Ed 2012; 97: 434-8.

12. Araki T, Sholl LM, Gerbaudo VH, et al. Thymic measurements in pathologically proven normal thymus and thymic hyperplasia: intraobserver and interobserver variabilities. Acad Radiol 2014; 21: 733-42.

13. McErlean A, Panicek DM, Zabor EC, et al. Intra- and interobserver variability in CT measurements in oncology. Radiology 2013; 269: 451-9. 